FOLIA

Amazónica

Revista del Instituto de Investigaciones

de la Amazonía Peruana

\title{
CARACTERIZACIÓN DE LA PESCA A PEQUEÑA ESCALA DEL RÍO TAHUAYO: BASES ECOLÓGICAS PARA UN MANEJO PESQUERO CON ENFOQUE DE CUENCA
}

\author{
Luis Carlos MOYA VÁSQUEZ ${ }^{1}$, Carlos Martín CAÑAS ALVA², Marina VARGAS \\ ROJAS $^{3}$, Emiliana ISASI-CATALA ${ }^{1}$
}

1 Wildlife Conservation Society - WCS. Calle Chiclayo 1008, Miraflores, Lima y Urbanización Sargento Lores Manzana Q, Lote 1, lquitos, Loreto - Perú.

2 Battelle. The National Ecological Observatory Network. Gainesville, Florida - USA

3 Instituto del Bien Común - IBC. Calle Nauta 334, Iquitos, Loreto - Perú

*Correo electrónico: Imoya@wcs.org

\section{RESUMEN}

La pesca a pequeña escala, como la que se desarrolla en la cuenca del río Tahuayo (CRT), ha sido poco estudiada a pesar de su importancia como suministro de proteína para la región. En este trabajo se presentan las principales características ecológicas de la pesca en la CRT a partir del análisis del desembarque pesquero. Entre agosto 2016 y julio 2017, se evaluó la riqueza y composición de especies, biomasa desembarcada y capturas por unidad de esfuerzo (CPUE), por periodos hidrológicos, ambientes acuáticos y comunidades. Las capturas presentaron 62 especies, agrupadas en 23 familias y 7 órdenes, siendo la familia Pimelodidae la más diversa (12 especies). La riqueza de especies fue muy similar entre períodos hidrológicos y entre ambientes acuáticos. El desembarque total ascendió a $13805,38 \mathrm{~kg}$, donde 17 especies representaron el 80,5\% de la biomasa. Más del $50 \%$ de la biomasa se desembarcó en transición a vaciante y vaciante, principalmente en la comunidad El Chino (71,5\%). La CPUE promedio anual para la CRT fue $0,51 \mathrm{~kg} / \mathrm{hp}(\mathrm{DE}=0,046)$, obteniéndose las capturas más bajas en temporada de creciente y en quebradas (KW, p-valor $<0,05$ ). Las especies más capturadas fueron las carachamas, los grandes bagres, el acarahuazú y el paco, siendo sus mayores capturas en vaciante. Los resultados muestran la importancia de considerar las dinámicas pesqueras de pequeña escala, bajo 
un enfoque de cuenca que considera un grupo diverso de peces, diferentes ambientes acuáticos y sus dinámicas a lo largo del ciclo hidrológico.

PALABRAS CLAVE: área de conservación regional, bosque inundable, comunidad ribereña, CPUE, Loreto, manejo pesquero.

\title{
CHARACTERIZATION OF SMALL-SCALE FISHERIES OF THE TAHUAYO RIVER: ECOLOGICAL BASES FOR FISHERIES MANAGEMENT WITH A BASIN APPROACH
}

\begin{abstract}
Small-scale fishing, such as that of the Tahuayo river basin (CRT) has been little studied despite its importance as a protein supply for the region. This article presents the fisheries' main ecological features in the CRT based on of an analysis of fish landings. Between August 2016 and July 2017, the richness and composition of species, biomass and catches per unit of effort (CPUE) were evaluated, by hydrological periods, aquatic environments and communities. The captures presented 62 species, grouped into 23 families and 7 orders, with the Pimelodidae family being the most diverse (12 species). Species richness was very similar between hydrological periods and between aquatic environments. The total landing amounted to $13,805.38 \mathrm{~kg}$, where 17 species represented $80.5 \%$ of the biomass. More than $50 \%$ of the biomass was landed in periods of low-water, mainly in the community of El Chino (71.5\%). The average annual CPUE for the CRT was $0.51 \mathrm{~kg} / \mathrm{hp}(\mathrm{DE}=0.046)$, obtaining the lowest catches in the high-water season and in streams (KW, p-value <0.05). The most captured species were the carachamas, the large catfish, the acarahuazú and the paco, with the highest catches being in the low-water season. All these results show the importance of considering small-scale fishing dynamics, under a basin approach that considers a diverse group of fish, different aquatic environments and their dynamics throughout the hydrological cycle.

KEYWORDS: Regional Conservation Area, floodable forest, riparian community, CPUE, Loreto, fisheries management.
\end{abstract}




\section{INTRODUCCIÓN}

Las medidas de manejo pesquero en la Amazonía peruana están basadas en la información generada por el seguimiento de los desembarques de la pesca comercial en los principales centros urbanos (capitales de provincia) (Cañas, 2000; Riofrio et al., 2008; García et al., 2012). Si bien estas localidades ofrecen las condiciones adecuadas para un registro diario de la pesca facilitando su caracterización y monitoreo (composición y volumen de las capturas, esfuerzo pesquero, variaciones temporales, entre otros), estos registros no siempre representan la dinámica de la pesca a pequeña escala, que es la que más predomina en la región Amazónica y que actualmente abastece de pescado a las principales ciudades (Almeida et al., 2009; García et al., 2009). De acuerdo con Berkes (2008), la pesquería de pequeña escala incluye la pesquería tradicional, la artesanal y la de subsistencia, predominando en países en vías de desarrollo como los Amazónicos. En este tipo de pesca se suele utilizar implementos tradicionales (pequeñas redes, trampas y cordeles), incluye una mayor variedad de especies y hace uso de stocks asociados a sistema de cochas del bosque inundable, con una riqueza y composición de especies que, por su bajo valor de venta, difieren de la pesca comercial (Almeida et al., 2009; Castello et al., 2013; Berkes et al., 2001). En su mayoría, esta actividad pesquera es realizada por comunidades ribereñas con acuerdos de pesca para un aprovechamiento directo y de subsistencia (Rodríguez et al., 2018). Esta modalidad de manejo es común en los ecosistemas inundables de la Amazonía (Crampton et al., 2004; Almeida et al., 2009; Castello et al., 2013), y es considerada clave no solo por tomar en cuenta el valioso conocimiento de las comunidades sobre los hábitats y ecología de los recursos pesqueros, sino también por la capacidad que tienen en adaptarse a la dinámica propia de los ecosistemas inundables (Crampton et al., 2004; Almeida et al., 2009; Rodríguez et al., 2018).

Si bien son pocos los estudios de la pesca a pequeña escala desde las mismas comunidades en Loreto (Anderson et al., 2009; García et al., 2012; Rodríguez et al., 2018), se sabe que en los últimos 30 años más del $70 \%$ el desembarque de pescado en la región fue abastecido por capturas provenientes desde las diferentes comunidades ribereñas ubicadas al interior de la región Loreto: 79 \% entre 1984-2006 (García et al., 2009), $79 \%$ entre 2008-2012 (García et al., 2012) y $69 \%$ entre 2016-2019 (DIREPRO-L, 2019). Asimismo, la pesca comercial proveniente de los grandes centros urbanos suele hacer uso de las zonas donde las comunidades ribereñas realizan la pesca a pequeña escala, lo que ha puesto en riesgo la sostenibilidad del aprovechamiento y generado conflictos entre pescadores foráneos (comerciales) y locales (Berkes et al., 2001; Chirif, 2012; Rodríguez et al., 2018; WCS, 2020). Esta realidad evidencia la importancia de conocer las características de la pesca a pequeña escala e incluirlas en futuras medidas de manejo pesquero de la región.

La actividad pesquera de la cuenca del río Tahuayo (CRT, Loreto, Perú) constituyela principal fuente de proteína animal en la dieta diaria de las comunidades establecidas dentro de la cuenca y un ingreso económico familiar importante para su subsistencia, la cual está basada en diferentes actividades de uso de recursos naturales del bosque inundable (Coomes, 1995; Smith et al., 2001; Chirif, 2012). La excesiva presión de extracción de la flota pesquera comercial de Iquitos impulsó a los habitantes del Tahuayo a organizarse para proponer estrategias de manejo de la pesca, basadas principalmente en la zonificación y en el control y vigilancia de los sistemas acuáticos utilizados en la extracción 
pesquera. Es así como, a finales de los años 90 $\mathrm{y}$, a partir de acuerdos comunales, tomaron el cuidado del sistema de cochas y otros cuerpos de agua que existe en sus bosques inundables a través de comités de control y vigilancia (Smith et al., 2001; Chirif, 2012). Estos acuerdos pesqueros no están reconocidos legalmente por la actual legislación pesquera, aun cuando estudios muestran que no ha habido indicios de sobrepesca en la cuenca (Summers \& Pinedo, 2001), por lo que este manejo podría ser clave para garantizar la sostenibilidad de la pesca en la CRT. Sin embargo, el aprovechamiento sostenible debe ser confirmado a partir de un seguimiento sistemático y análisis de la actividad pesquera con un enfoque de cuenca. Esta evaluación del desembarque de pescado en la CRT provee información de la actividad pesquera y su relación con los ambientes acuáticos y las condiciones hidrológicas naturales de un bosque inundable de la Amazonía peruana, como base para proponer recomendaciones de manejo pesquero con un enfoque de cuenca.

\section{MATERIAL Y MÉTODOS}

\section{ÁREA DE ESTUDIO}

El río Tahuayo es un tributario de aguas negras ubicado en la margen derecha del río Amazonas (Figura 1), a $65 \mathrm{~km}$ al sur de la ciudad de Iquitos,

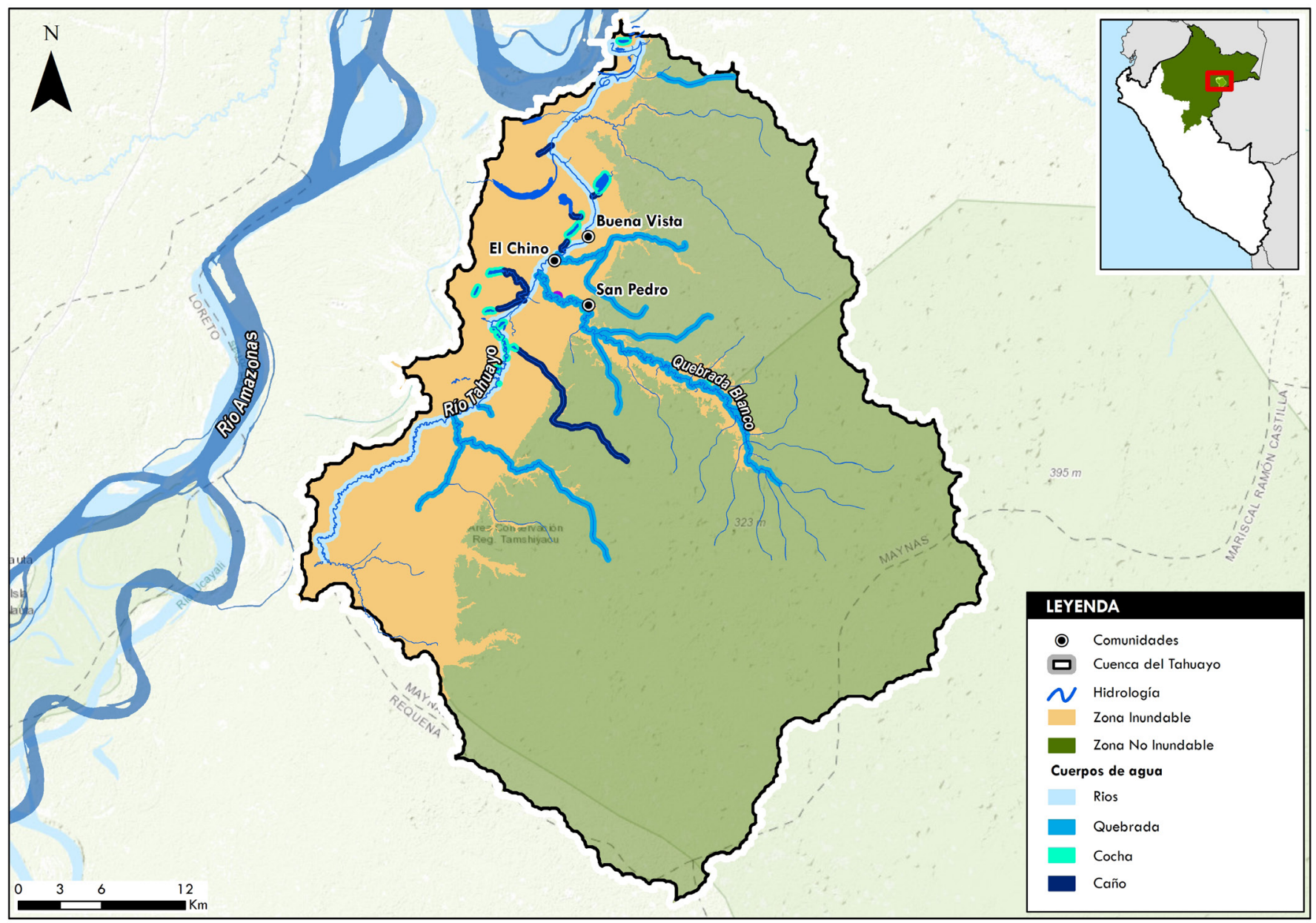

Figura 1. La cuenca del río Tahuayo y sus ambientes acuáticos relacionados al río Amazonas, en Loreto (Perú). 
principal centro comercial en la región Loreto (Perú). Comprende un área de drenaje de $1400 \mathrm{~km}^{2}$, que se caracteriza por la presencia de un bosque de colinas bajas en su lado este (asociadas a la Quebrada Blanco, su único tributario) y por un extenso bosque aluvial inundable y pantano de palmeras asociados a un sistema de tahuampas, restingas y lagunas (cochas) en el lado oeste.

En la CRT, las acciones de manejo de recursos naturales que han venido realizando por más de 10 años comunidades como San Pedro, El Chino y Buena Vista, han logrado el establecimiento de acuerdos de aprovechamiento comunal y la creación del Área de Conservación Regional Comunal Tamshiyacu Tahuayo (ACRCTT, 2010). Las comunidades basan su subsistencia, principalmente en pesca, caza y agricultura (Coomes, 1992; McDaniels, 1997; Flores et al., 2016), siendo la pesca una actividad a pequeña escala, manejada a partir de acuerdos comunales en los que se prohíbe el ingreso de pescadores comerciales, se ordenan el uso de cochas y otros cuerpos de agua (protección, recuperación y de subsistencia o comercial), se establecen cuotas de aprovechamiento, uso de mallas con abertura de malla mayor a 2" y se establece un sistema comunal para vigilar y controlar el acceso a las zonas de pesca. La comunidad El Chino cuenta con una Asociación de Pescadores Artesanales (Puma Garza I), con 27 socios acreditados como pescadores artesanales de la región y reconocida por el Ministerio de la Producción (Vargas, 2017).

\section{AMBIENTES ACUÁTICOS Y PERIODOS HIDROLÓGICOS EN LA CUENCA DEL RÍO TAHUAYO}

Se consideraron cuatro categorías de ambientes acuáticos para clasificar las zonas comúnmente utilizadas para la pesca por los pescadores de las comunidades de la CRT: río (R: ambiente de flujo que recibe agua de tributarios y de los sistemas de cochas en la cuenca), quebrada (Q: ambiente de flujo que desemboca en el río principal y nace en el bosque de tierra firme no inundable), caños (A: ambiente de bajo flujo que conectan las cochas con el canal principal del río) y cocha (C: cuerpo de agua léntico adyacente al canal principal del río).

El régimen hidrológico de la CRT es controlado por el río Amazonas, que durante la época de lluvias inunda el lado oeste de la cuenca e incrementa el nivel de agua entre 6-8 metros, dejando sumergidos extensos bosques y permitiendo el ingreso de peces a todo el sistema de cochas, quebradas y caños (Coomes, 1995; Smith et al., 2001). A partir de los datos de caudal de la estación Tamshiyacu (Espinoza et al., 2009) y nivel de agua de la estación Iquitos del Servicio de Hidrografía y Navegación de la Amazonía (Marina de Guerra del Perú, 2018), se asumen 4 estaciones hidrológicas para la cuenca: época de vaciante (agosto a octubre), época de transición a creciente (noviembre a enero), época de creciente (febrero a abril) y época de transición a vaciante (mayo a julio).

\section{REGISTRO DEL DESEMBARQUE Y ANÁLISIS DE DATOS}

Los desembarques de pescado fueron registrados diariamente de 6:00 a 9:30 am y 3:30 a 5:30 pm (horarios habituales de pesca de las comunidades), por pescadores de las tres comunidades de la CRT (Figura 1). Estos pescadores fueron seleccionados en asamblea comunal y fueron capacitados y acompañados por una de las autoras de este artículo (M. Vargas). El periodo de registro comprendió el año hidrológico 2016-2017, desde agosto 2016 (vaciante) a julio 2017 (transición a vaciante). En cada desembarque se registró: fecha, estación hidrológica (vaciante: $\mathrm{V}$, transición a creciente: $\mathrm{tC}$, creciente: $\mathrm{C}$ y transición a vaciante: $\mathrm{tV}$ ), hora de inicio y final de la pesca, pescador, comunidad 
de origen, especies, volumen en kilogramos por especie, zona de pesca, ambiente acuático y uso de la pesca (consumo o venta). La identificación de las especies se realizó utilizando la lista anotada de especies ictiológicas propuesta por Ortega et al. (2011) y registros fotográficos revisados por especialistas del Museo de Historia Natural de la Universidad Nacional Mayor de San Marcos. Se calculó la riqueza y composición de especies desembarcadas para toda la cuenca, así como la biomasa (kilogramos de captura por especie) considerando periodos hidrológicos, ambientes acuáticos y comunidades. Se estimaron las capturas por unidad de esfuerzo (CPUE, kilogramos de captura por tiempo efectivo de pesca por pescador $=\mathrm{kg} / \mathrm{hp}$ ) total $\mathrm{y}$ por especie, y se evaluaron las diferencias entre periodos hidrológicos, ambientes acuáticos $\mathrm{y}$ comunidades pesqueras, a partir de pruebas no paramétricas de Kruskal-Wallis y pruebas post hoc de Mann-Whitney ( $\alpha \geq 0.05$ ), utilizando Past 4.05 (Hammer et al., 2001).

\section{RESULTADOS}

En total se identificaron 43 zonas de pesca (ZP) utilizadas por 83 pescadores de la CRT, siendo las cochas los ambientes acuáticos con más cantidad de ZP (24), seguidas por las quebradas (10), caños (8) y el río (1). La composición de las capturas estuvo representada por 62 especies, agrupadas en 23 familias y 7 órdenes (Tabla 1). La familia Pimelodidae tuvo la mayor riqueza de especies desembarcadas (12), seguida de las familias Cichlidae (7), Serrasalmidae (5) y Curimatidae (5), siendo el orden Characiformes el más diverso (30). Los pescadores agrupan estas especies bajo 53 nombres comunes, cinco de estos nombres incluyeron varias especies de una misma familia: sábalos (Bryconidae, 2 especies), lisas (Anostomidae, 3 especies), pañas (Serrasalmidae, 3 especies), shuyos (Erythrinidae, 2 especies) y bujurquis (Cichlidae, 4 especies). La riqueza de especies desembarcadas fue muy similar entre períodos hidrológicos $(\mathrm{C}=47, \mathrm{tV}=47, \mathrm{~V}=46 \mathrm{y}$ $\mathrm{tC}=45$ especies) y entre ambientes acuáticos $(\mathrm{R}=48$. $\mathrm{Q}=49, \mathrm{~A}=46$ y $\mathrm{C}=48$, Tabla 1). Especies como el dentón, panshina, macana y zúngaro alianza fueron desembarcadas en $\mathrm{C} y \mathrm{tV}$ únicamente, mientras otras como el chio chio, san pedro, pez lápiz e insimiracú fueron desembarcadas en $\mathrm{V}$ y tC. Las especies panshina, cunchimama y zúngaro alianza provienen principalmente de quebradas, mientras que el chío chio provino principalmente de cochas.

El desembarque total registrado entre 20162017 en las tres comunidades de la CRT ascendió a $13805,38 \mathrm{~kg}$. Considerando las nominaciones locales, 17 especies representaron el 80,5 \% de la biomasa del desembarque anual, siendo las más importantes el fasaco (12,8\%), la doncella $(9,5 \%)$, la carachama $(7,4 \%)$, el acarahuazú (6\%), la palometa (5,9\%) y el sábalo (5,5\%). Las 36 especies restantes representaron el $20 \%$ del total, ninguna con más del $2 \%$ de representatividad en el desembarque anual. La mayor biomasa la presentaron los órdenes Characiformes (48,8 \%) y Siluriformes (34,7 \%). Más de la mitad de la biomasa se desembarcó en transición a vaciante $(31,7 \%)$ y vaciante (26,8\%), alcanzando el máximo en julio 2017 $(2269,9 \mathrm{~kg})$. El periodo de transición a creciente registró solo el 16,8 \% de la biomasa total, con el mínimo desembarcado en diciembre 2017 (317,1 kg) (Figura 2). La mayor parte de la captura fue desembarcada en El Chino (71,5\%) por 56 pescadores, seguida por Buena Vista (15\%) con 16 pescadores y San Pedro (13,5 \%) con 11 pescadores. Buena Vista destinó la mayor parte de su captura al consumo (88,6 \%), mientras que los pescadores de San Pedro y El Chino dedicaron la captura en partes aproximadamente iguales para la venta $(42,9$ y $55,6 \%$, respectivamente) y consumo $(57,1 \%$ y 44,4 \%, respectivamente). 
Tabla 1. Lista de especies de consumo, volumen desembarcado y porcentaje del volumen representado por cada especie en la CRT, considerando el período hidrológico y ambiente acuático de la pesca.

\begin{tabular}{|c|c|c|c|c|c|c|c|}
\hline Orden & Familia & Nombre científico & $\begin{array}{l}\text { Nombre } \\
\text { común }\end{array}$ & $\mathrm{VT}(\mathrm{kg})$ & PVE (\%) & $\mathrm{PH}$ & $A C$ \\
\hline Osteoglossiformes & Osteoglossidae & $\begin{array}{l}\text { Osteoglossum } \\
\text { bicirrhosum }\end{array}$ & Arahuana & 79,4 & 0,6 & $C+V \vee+C$ & A R C \\
\hline Clupeiformes & Pristigasteridae & Pellona sp. & Panshina & 2,6 & 0,0 & tV & QR \\
\hline \multirow{25}{*}{ Characiformes } & Acestrorhynchidae & Acestrorhynchus sp. & Cashorro & 171,1 & 1,2 & $C+V \vee+C$ & $A Q R C$ \\
\hline & Anostomidae & $\begin{array}{l}\text { Schizodon fasciatus } \\
\text { Rhytiodus microlepis } \\
\text { Leporinus sp. }\end{array}$ & Lisa* & 287,5 & 2,1 & $C+V \vee+C$ & $A Q R C$ \\
\hline & Bryconidae & $\begin{array}{l}\text { Brycon amazonicus } \\
\text { Brycon melanopterus }\end{array}$ & Sábalo* & 765,9 & 5,5 & $C+V \vee t C$ & $A Q R C$ \\
\hline & Chalceidae & Chalceus erythrurus & San pedro & 6,5 & 0,0 & $\mathrm{~V}+\mathrm{C}$ & $A Q R C$ \\
\hline & \multirow{2}{*}{ Characidae } & Moenkhausia sp. & Mojara & 12,3 & 0,1 & $\mathrm{CtV}$ & $A Q C$ \\
\hline & & Roeboides myersii & Denton & 2,2 & 0,0 & $C+V \vee+C$ & $A Q R$ \\
\hline & Ctenoluciidae & Boulengerella maculata & Pez lápiz & 8,4 & 0,1 & $\mathrm{VtC}$ & $A Q R C$ \\
\hline & \multirow{5}{*}{ Curimatidae } & Curimata vittata & Insimiracu & 1,0 & 0,0 & tC & C \\
\hline & & $\begin{array}{l}\text { Potamorhina } \\
\text { altamazonica }\end{array}$ & Llambina & 249,9 & 1,8 & $\mathrm{VtC}$ & $A C$ \\
\hline & & Potamorhina latior & Yahuarachi & 193,3 & 1,4 & $C+V \vee t C$ & $A Q R C$ \\
\hline & & Psectrogaster amazonica & Ractacara & 41,0 & 0,3 & $C+V \vee+C$ & $A Q R C$ \\
\hline & & Psectrogaster rutiloides & Chio chio & 2,0 & 0,0 & $C+V \vee+C$ & $A Q R C$ \\
\hline & \multirow{2}{*}{ Cynodontidae } & Hydrolicus scomberoides & Huapeta & 20,8 & 0,2 & $C+V \vee t C$ & $A Q R C$ \\
\hline & & Rhapiodon vulpinus & Chambira & 194,3 & 1,4 & $C+V \vee t C$ & $A Q R C$ \\
\hline & \multirow[t]{2}{*}{ Erythrinidae } & $\begin{array}{l}\text { Erythrinus erythrinus } \\
\text { Hoplerythrinus } \\
\text { unitaeniatus } \\
\end{array}$ & Shuyo* & 288,2 & 2,1 & $C+V \vee+C$ & $A Q R C$ \\
\hline & & Hoplias malabaricus & Fasaco & 1761,3 & 12,8 & $C+V \vee+C$ & $A Q R C$ \\
\hline & Hemiodontidae & Anodus elongatus & Yulilla & 8,4 & 0,1 & $\mathrm{CV}$ & A R C \\
\hline & \multirow{2}{*}{ Prochilodontidae } & Prochilodus nigricans & Boquichico & 532,5 & 3,9 & $C+V \vee+C$ & $A Q R C$ \\
\hline & & Semaprochilodus insignis & Yaraqui & 12,3 & & $C+V \vee t C$ & $A Q R C$ \\
\hline & \multirow{5}{*}{ Serrasalmidae } & Colossoma macropomum & Gamitana & 38,7 & 0,3 & $C+V \vee+C$ & $A Q R C$ \\
\hline & & Myleus rubripinnis & Curuhuara & 248,7 & 1,8 & $C+V \vee+C$ & QRC \\
\hline & & Mylossoma albiscopum & Palometa & 811,9 & 5,9 & $C+V \vee+C$ & $A Q R C$ \\
\hline & & Piaractus brachypomus & Paco & 545,9 & 4,0 & $C+V \vee t C$ & $A Q R C$ \\
\hline & & $\begin{array}{l}\text { Serrasalmus humeralis } \\
\text { Serrasalmus spilopleura } \\
\text { Pygocentrus nattereri }\end{array}$ & Paña* & 472,2 & 3,4 & $C+V \vee+C$ & $A Q R C$ \\
\hline & Triportheidae & Triportheus elongatus & Sardina & 67,2 & 0,5 & $C+V \vee t C$ & $A Q R C$ \\
\hline Gymnotiformes & Apteronotidae & $\begin{array}{l}\text { Sternarchorhynchus } \\
\text { oxyrhynchus }\end{array}$ & Macana & 7,3 & 0,1 & $\mathrm{CtV}$ & QRC \\
\hline
\end{tabular}




\begin{tabular}{|c|c|c|c|c|c|c|c|}
\hline \multirow{20}{*}{ Siluriformes } & \multirow[b]{2}{*}{ Auchenipteridae } & Ageneiosus sp. & Bocon & 270,4 & 2,0 & $C+V \vee+C$ & $A Q R C$ \\
\hline & & $\begin{array}{l}\text { Parauchenipterus } \\
\text { galeatus }\end{array}$ & Novia & 87,7 & 0,6 & $C+V \vee+C$ & $A Q R C$ \\
\hline & Callichthyidae & Hoplosternum littorale & Shiruy & 12,8 & 0,1 & $\mathrm{C}+\mathrm{tV} \mathrm{tC}$ & $A Q R C$ \\
\hline & \multirow{3}{*}{ Doradidae } & $\begin{array}{l}\text { Megalodoras } \\
\text { uranoscopus }\end{array}$ & Churero & 146,5 & 1,1 & $C+V \vee+C$ & $A Q R C$ \\
\hline & & Oxydoras niger & Turushuqui & 196,8 & 1,4 & $\mathrm{C}+\mathrm{V}+\mathrm{C}$ & $\mathrm{A} Q \mathrm{RC}$ \\
\hline & & Pterodoras granulosus & Cahuara & 182,6 & 1,3 & $\mathrm{C}+\mathrm{V} \vee \mathrm{tC}$ & $A Q R C$ \\
\hline & \multirow[t]{2}{*}{ Loricariidae } & $\begin{array}{l}\text { Pseudorinelepis } \\
\text { genibarbis }\end{array}$ & $\begin{array}{l}\text { Carachama } \\
\text { chica }\end{array}$ & 431,8 & 3,1 & $C+V \vee+C$ & $A Q R C$ \\
\hline & & Pterigoplichthys pardalis & Carachama & 1016,5 & 7,4 & $C+V \vee t C$ & $A Q R C$ \\
\hline & \multirow{12}{*}{ Pimelodidae } & $\begin{array}{l}\text { Brachyplatystoma } \\
\text { juruense }\end{array}$ & $\begin{array}{l}\text { Zungaro } \\
\text { alianza }\end{array}$ & 1,0 & 0,0 & $C+V \vee t C$ & QR C \\
\hline & & Callophysus macropterus & Mota & 34,4 & 0,2 & $C+V \vee+C$ & $A Q R C$ \\
\hline & & Cheirocerus sp & Cunchi & 14,3 & 0,1 & $C \vee$ & $A Q R$ \\
\hline & & $\begin{array}{l}\text { Hemisorubim } \\
\text { platyrhynchos }\end{array}$ & Toa & 18,3 & 0,1 & $C+V \vee+C$ & $A Q$ \\
\hline & & Hypopthalmus edentatus & Maparate & 31,0 & 0,2 & $C+V \vee t C$ & $A Q R C$ \\
\hline & & Leiarus marmoratus & Achara & 113,2 & 0,8 & $C+V \vee t C$ & $A Q R C$ \\
\hline & & $\begin{array}{l}\text { Phractocephalus } \\
\text { hemiliopterus }\end{array}$ & Pez torre & 33,1 & 0,2 & $C+V \vee+C$ & $A Q R C$ \\
\hline & & Pimelodus blocchii & Bagre & 77,2 & 0,6 & $C+V \vee+C$ & QRC \\
\hline & & $\begin{array}{l}\text { Pseudoplatystoma } \\
\text { punctifer }\end{array}$ & Doncella & 1312,9 & 9,5 & $C+V V$ & $A Q R C$ \\
\hline & & $\begin{array}{l}\text { Pseudoplatystoma } \\
\text { tigrinum }\end{array}$ & $\begin{array}{l}\text { Tigre } \\
\text { zungaro }\end{array}$ & 327,2 & 2,4 & $C+V \vee t C$ & $A Q R C$ \\
\hline & & Sorubim lima & Shiripira & 10,0 & 0,1 & $\mathrm{CtV} V$ & $A Q R C$ \\
\hline & & Zungaro zungaro & Cunchimama & 469,4 & 3,4 & tV & Q \\
\hline \multirow{4}{*}{ Cichliformes } & \multirow{4}{*}{ Cichlidae } & $\begin{array}{l}\text { Aequidens tetramerus } \\
\text { Bujurquina apoparuana } \\
\text { Hypselacara temporalis } \\
\text { Acaronia nasa }\end{array}$ & Bujurqui* & 458,5 & 3,3 & $C+V \vee+C$ & $A Q R C$ \\
\hline & & Astronotus ocellatus & Acarahuazu & 833,2 & 6,0 & $C+V \vee+C$ & $A Q R C$ \\
\hline & & Cichla monoculus & Tucunaré & 501,1 & 3,6 & $C+V \vee+C$ & $A Q R C$ \\
\hline & & Crenicichla sp. & Añashua & 100,8 & 0,7 & $C+V \vee+C$ & $A Q R C$ \\
\hline Perciformes & Sciaenidae & $\begin{array}{l}\text { Plagioscion } \\
\text { squamosissimus }\end{array}$ & Corvina & 292,3 & 2,1 & $C+V \vee+C$ & $A Q R C$ \\
\hline
\end{tabular}

VT: volumen desembarcado (kg), PVE: porcentaje del volumen representado por cada especie (\%), PH: período hidrológico, AC: ambiente acuático, V: vaciante, tC: transición a creciente, C: creciente y tV: transición a vaciante, R: río, Q: quebrada, A: caño y C: cocha. Se resaltan las especies con mayores volúmenes de desembarco en la CRT

*Nombre local que agrupa varias especies 


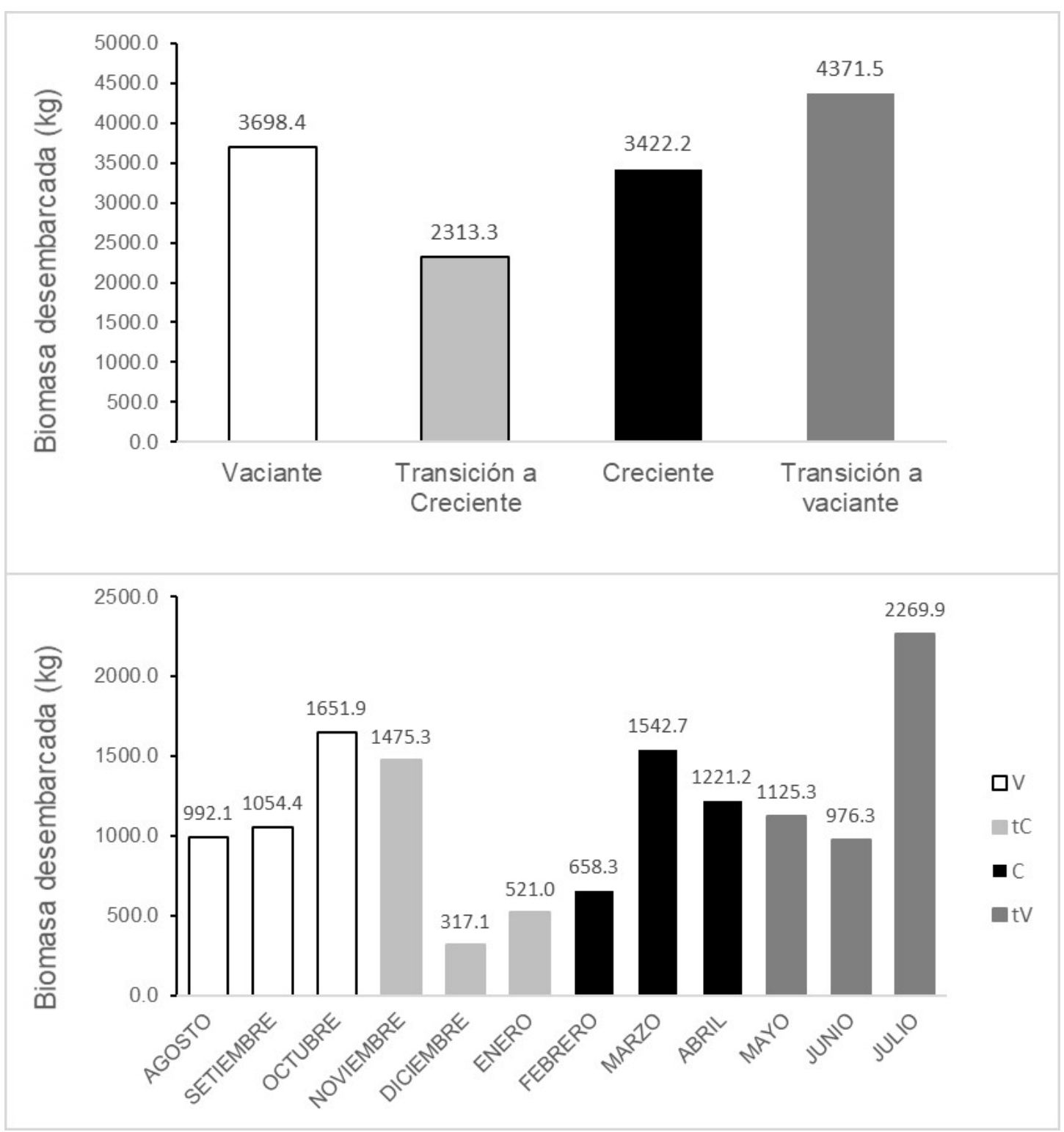

Figura 2. Biomasa $(\mathrm{kg})$ de pescado desembarcada en la cuenca del río Tahuayo durante cada estación hidrológica (V: vaciante, tC: transición a creciente, C: creciente y tV: transición a vaciante) y mensual (agosto 2016 - julio 2017). 
La CPUE promedio anual para la CRT fue 0,51 kg/hp (DE 0,046, Tabla 2), siendo muy variable, presentando valores mínimos de 0,06 $\mathrm{kg} / \mathrm{hp}$ y máximos de 2,50 kg/hp. Las CPUE fueron diferentes entre temporadas del período hidrológico (KW H=49,63; $\mathrm{n}=195 ; \mathrm{p}$-valor $<0,05)$, principalmente para creciente (Figura 3), temporada en el que se registraron los valores más bajos de CPUE $(0,26 \mathrm{~kg} / \mathrm{hp}$; DE 0,28; MW p-valor $<0,05)$. Para el resto de las temporadas los valores fueron similares (MW p-valor >0,05; excepto entre $\mathrm{V} y \mathrm{tV}$ ), siendo máximo en vaciante $(0,1 \mathrm{~kg} / \mathrm{hp} ; \mathrm{DE} 0,68)$. Las capturas también fueron diferentes entre ambientes acuáticos (KW H=18,41; $n=191 ; \mathrm{p}$-valor <0,05, Tabla 2), principalmente para las quebradas, en donde las capturas resultaron bajas en comparación al resto de los ambientes de pesca $(0,28 \mathrm{~kg} /$ hp; DE 0,21; MW p-valor <0,05, Figura 3). Para todos los ambientes acuáticos, las CPUE fueron diferentes entre temporadas hidrológicas (R KW $\mathrm{H}=9,86$; $\mathrm{n}=106$; p-valor $<0,05$; Q KW $\mathrm{H}=22,97$; $\mathrm{n}=88$; p-valor <0,05; A KW H=9,75; $\mathrm{n}=61$; p-valor $<0,05$; C KW H=10,47; n=94; p-valor <0,05, Tabla 2), siendo las temporadas de creciente (CPUE entre 0,19 y $0,43 \mathrm{~kg} / \mathrm{hp}$ ) y vaciante (CPUE entre 0,59 a 1,10 kg/hp) las de mayor contraste (MW p-valor $<0,05$ ).

La comunidad con mayor CPUE de pesca fue El Chino $(0,57 \mathrm{~kg} / \mathrm{hp}$; DE=0,61; KW H=12,19; $\mathrm{n}=83$; p-valor $<0,05$, Tabla 2), seguida de Buena Vista $(0,31 \mathrm{~kg} / \mathrm{hp} ; \mathrm{DE}=0,30)$ y San Pedro $(0,29$ $\mathrm{kg} / \mathrm{hp}$; $\mathrm{DE}=0,18$ ), siendo la intensidad de la pesca entre estas dos últimas similares entre sí (MW p-valor $<0,05$, Figura 3). Las CPUE de todas las comunidades resultaron variables según el período hidrológico (BV KW $\mathrm{H}=19,76 ; \mathrm{n}=48$; p-valor <0,05; CH KW H=20,82; n=114; p-valor $<0,05$; SP KW $\mathrm{H}=19,00 ; \mathrm{n}=31$; p-valor $<0,05$, Tabla 2). La creciente fue la temporada con menos CPUE de pesca para las tres comunidades (BV 0,20 DE 0,16; CH 0,30 DE 0,34; SP 0,19 DE
$0,14)$ presentándose los máximos valores en vaciante (BV 0,74 DE 0,37; CH 0,69 DE 0,52; SP 1,60 DE 1,33, KW p-valor <0.05, MW p-valor $<0.05$, Tabla 2). Para Buena Vista y San Pedro las CPUE de pesca resultó similar entre ambientes acuáticos (BV KW $\mathrm{H}=8,08 ; \mathrm{n}=42$; $\mathrm{p}$-valor >0,05; SP KW H=0,05; $n=16$; p-valor $>0,05$, Tabla 2). Sin embargo, la pesca registrada en El Chino resultó menor en quebradas (CPUE 0,20 DE 0,18), que en el resto de los ambientes acuáticos ( $\mathrm{CH} \mathrm{KW}$ $H=22$,28; $n=133$; $p$-valor $<0,05$, Tabla 2), siendo las cochas los ecosistemas más importantes (CPUE 1,56 DE 2,33; MW p-valor <0,05).

Las CPUE de las 17 principales especies muestran que existen diferencias en los patrones de aprovechamiento registrados (KW $\mathrm{H}=124,1$; $\mathrm{n}=687$; p-valor $<0,05$, Tabla 3). Las dos especies de carachama (carachama chica 1,66 kg/hp DE 3,41; carachama $1,23 \mathrm{~kg} / \mathrm{hp}$ DE 2,13), los tres grandes bagres (cunchimama 1,49 kg/hp DE 1,20; doncella $1,26 \mathrm{~kg} / \mathrm{hp}$ DE 1,18 y tigre zúngaro $0,88 \mathrm{~kg} / \mathrm{hp}$ DE 0,97 ), el acarahuazú y paco son las especies más aprovechadas. Mientras que las lisas $(0,24 \mathrm{~kg} / \mathrm{hp}$ DE 0,24$)$ y las pañas $(0,19$ $\mathrm{kg} / \mathrm{hp} \mathrm{DE} \mathrm{0,19),} \mathrm{que} \mathrm{agrupan} \mathrm{a} \mathrm{varias} \mathrm{especies,}$ obtuvieron los valores más bajos de CPUE (MW p-valor $<0,05$ ).

Las CPUE de las 10 especies con más volúmenes de aprovechamiento fue variable en el ciclo hidrológico (Tabla 3), excepto para acarahuazú, palometa, sábalo, paco y pañas (KW p-valor $>0,05$, Figura 4). Para el resto de las especies, las mayores CPUE se obtuvieron en el periodo de vaciante y la transición a creciente CPUE (MW p-valor $<0,05$ ), siendo el período de creciente el que presentó las CPUE más bajas. Para la mayoría de estas especies las CPUE fueron similares entre los diferentes ambientes acuáticos (KW p-valor $>0,05$, Tabla 3, Figura 5), excepto para el fasaco, sábalo y boquichico, cuyas CPUE fueron menores en quebradas (fasaco 0,28 kg/hp DE 0,38; sábalo $0,29 \mathrm{~kg} / \mathrm{hp} \mathrm{DE} \mathrm{0,22;} \mathrm{boquichico} \mathrm{0,18} \mathrm{kg/hp}$ 
DE 0,22 ) que en relación a los otros ambientes acuáticos (MW p-valor $<0,05$ ). Las CPUE de estas especies fue similar entre las tres comunidades de la CRT (KW p-valor $<0,05$, Tabla 3), sólo la carachama (KW $\mathrm{H}=8,74 ; \mathrm{n}=41$; $\mathrm{p}$-valor $<0,05$ ) y la palometa (KW $\mathrm{H}=7,02 ; \mathrm{n}=50$; $\mathrm{p}$-valor $<0,05$ ) presentaron CPUE diferentes entre comunidades, siendo la comunidad de El Chino la que presentó mayores CPUE para estas especies (carachama 1,66 kg/hp DE 2,46; palometa 0,35 kg/hp DE 0,39 ), mientras que Buena Vista la de menor captura de carachama $(0,31 \mathrm{~kg} / \mathrm{hp}$ DE 0,32$)$ y San Pedro la de palometa $(0,09 \mathrm{~kg} / \mathrm{hp}$ DE 0,04$)$.

\section{DISCUSIÓN}

La riqueza y composición de especies desembarcadas en la CRT evidenció una pesca dirigida a un grupo diverso de peces (Tabla 1), donde 17 especies representaron el $80 \%$ de la biomasa desembarcada en el periodo evaluado, representando una gran variedad de grupos tróficos: omnívoros, piscívoros y detritívoros. En Iquitos el $80 \%$ del desembarque anual estuvo representado por 13 especies, de las cuales tres (boquichico, palometa y llambina) representaron el $47 \%$ (DIREPRO-L, 2016; DIREPRO-L, 2017), siendo estas especies detritívoras las que han dominado los desembarques comerciales en Loreto en los últimos 30 años (García-Dávila et al., 2018). Sin embargo, en la CRT estas tres especies representaron solo el 11,5\% del desembarque anual evaluado.

Destaca la importancia del fasaco y la carachama para la CRT, aun cuando no figuran como especies relevantes en las estadísticas comerciales de Loreto (DIREPRO-L, 2016; DIREPRO-L, 2017). Recién desde el año 2011 el fasaco ha comenzado a registrarse en mayores volúmenes en la ciudad de Iquitos, particularmente en estado seco-salado (García et al., 2009; García-Dávila et al., 2018). En otras zonas inundables del río Amazonas los

Vol. 29 (1) 2020. 371-390 registros comerciales destacan la importancia de la carachama (Cerdeira et al., 2000).

Junto a los órdenes Characiformes y Siluriformes, el orden Cichliformes, representado por la familia Cichlidae, resultó de gran importancia para la CRT (Tabla 1), no sólo por la riqueza (7 especies), sino por la biomasa desembarcada $(13,7 \%)$ y CPUE (e.g. acarahuazú fue la sexta especie con mayores CPUE), aun cuando en los desembarques algunas especies fueron registradas bajo un mismo nombre local: bujurqui. Resultados similares se han reportado en las cuencas de los ríos Ampiyacu y Apayacu en la región Loreto (Rodríguez et al., 2018). Estos resultados contrastan con los reportes de pesca comercial, en los que este orden ha sido ubicado muy por debajo de los Characiformes y Siluriformes, y en algunos años inclusive al orden Osteoglossiformes (que agrupa a Arapaima gigas y Osteoglossum bicirrhosum), no sólo en la región de Loreto (García et al., 2009), sino en otras regiones como Madre de Dios (Cañas, 2000). Estos resultados permiten evidenciar las diferencias entre la composición de las capturas reportadas en los terminales pesqueros y las que se explotan en la CRT o en las comunidades ribereñas en Loreto, muchas de las cuales, como las ubicadas en los ríos Ucayali y Amazonas, abastecen de pescado a la ciudad de Iquitos (García et al., 2009). Es así como se evidencia la importancia de incluir en el manejo pesquero a especies consideradas de menor importancia en la pesca comercial, pero de gran relevancia para las comunidades ribereñas de la región Loreto. Por otra parte, es necesario incrementar el esfuerzo de conocer y monitorear la actividad pesquera de pequeña escala en las mismas zonas de extracción, como en la CRT o localidades como San Pablo de Tipishca (ruta Nauta a Yurimaguas) o Carocurahuayte (ruta Requena a Iquitos), desde donde las comunidades ribereñas envían sus capturas en embarcaciones de carga y pasajeros hacia la ciudad de Iquitos (García et al., 2009). 
Tabla 2. Promedio de captura por unidad de esfuerzo general, por período hidrológico, ambiente acuático y comunidades de la cuenca del río Tahuayo.

\begin{tabular}{|c|c|c|c|c|c|c|c|c|c|c|c|c|c|}
\hline \multicolumn{14}{|c|}{ CPUE promedio (Pr, kg/hp) y desviación estándar } \\
\hline & & \multirow{2}{*}{ Anual } & \multicolumn{4}{|c|}{$\mathrm{PH}$} & & & \multirow{2}{*}{ Anual } & \multicolumn{4}{|c|}{$\mathrm{PH}$} \\
\hline & & & $\mathrm{V}$ & tC & $\mathrm{C}$ & tV & & & & V & tC & C & tV \\
\hline & $\operatorname{Pr}$ & 0,51 & 0,81 & 0,69 & 0,26 & 0,45 & \multicolumn{7}{|c|}{ CM } \\
\hline & DE & 0,46 & 0,68 & 0,81 & 0,28 & 0,48 & BV & $\operatorname{Pr}$ & 0,31 & 0,74 & 0,78 & 0,20 & 0,24 \\
\hline & & & $A C$ & & & & & DE & 0,30 & 0,37 & 1,31 & 0,16 & 0,28 \\
\hline \multirow[t]{2}{*}{$\mathrm{R}$} & $\operatorname{Pr}$ & 0,58 & 0,59 & 0,56 & 0,43 & 0,55 & $\mathrm{R}$ & $\operatorname{Pr}$ & 0,43 & 0,63 & 0,57 & 0,51 & 0,36 \\
\hline & DE & 0,43 & 0,26 & 0,35 & 0,44 & 0,57 & & $\mathrm{DE}$ & 0,31 & 0,30 & 0,41 & 0,57 & 0,36 \\
\hline \multirow[t]{2}{*}{ Q } & $\operatorname{Pr}$ & 0,28 & 1,10 & 0,75 & 0,21 & 0,24 & $Q$ & $\operatorname{Pr}$ & 0,16 & - & - & 0,21 & 0,01 \\
\hline & DE & 0,21 & 1,07 & 0,78 & 0,17 & 0,21 & & $\mathrm{DE}$ & 0,28 & - & - & 0,32 & \\
\hline \multirow[t]{2}{*}{ A } & $\operatorname{Pr}$ & 0,63 & 0,90 & 0,70 & 0,37 & 0,68 & $A$ & $\operatorname{Pr}$ & 0,23 & - & 0,09 & 0,17 & 0,30 \\
\hline & DE & 0,78 & 0,61 & 0,90 & 0,36 & 1,12 & & $\mathrm{DE}$ & 0,19 & - & 0,00 & 0,11 & 0,30 \\
\hline \multirow[t]{18}{*}{ C } & $\operatorname{Pr}$ & 0,76 & 0,73 & 0,62 & 0,19 & 0,82 & C & $\operatorname{Pr}$ & 0,33 & 0,86 & 0,87 & 0,18 & 0,16 \\
\hline & DE & 1,10 & 0,57 & 0,99 & 0,14 & 1,41 & & $\mathrm{DE}$ & 0,39 & 0,48 & 1,47 & 0,15 & 0,19 \\
\hline & & & & & & & $\mathrm{CH}$ & $\operatorname{Pr}$ & 0,57 & 0,69 & 0,59 & 0,30 & 0,64 \\
\hline & & & & & & & & $\mathrm{DE}$ & 0,61 & 0,52 & 0,60 & 0,34 & 0,54 \\
\hline & & & & & & & $\mathrm{R}$ & $\operatorname{Pr}$ & 0,49 & 0,56 & 0,52 & 0,33 & 0,43 \\
\hline & & & & & & & & $\mathrm{DE}$ & 0,37 & 0,30 & 0,18 & 0,26 & 0,47 \\
\hline & & & & & & & $Q$ & $\operatorname{Pr}$ & 0,20 & - & - & 0,24 & 0,17 \\
\hline & & & & & & & & DE & 0,18 & - & - & 0,22 & 0,18 \\
\hline & & & & & & & $A$ & $\operatorname{Pr}$ & 1,05 & 0,90 & 2,21 & 0,75 & 0,82 \\
\hline & & & & & & & & $\mathrm{DE}$ & 0,67 & 0,54 & 1,42 & 0,82 & 0,40 \\
\hline & & & & & & & C & $\operatorname{Pr}$ & 1,56 & 0,75 & 0,39 & - & 2,82 \\
\hline & & & & & & & & $\mathrm{DE}$ & 2,33 & 0,82 & - & - & 3,16 \\
\hline & & & & & & & SP & $\operatorname{Pr}$ & 0,29 & 1,60 & 0,89 & 0,19 & 0,14 \\
\hline & & & & & & & & $\mathrm{DE}$ & 0,18 & 1,33 & 0,60 & 0,14 & 0,10 \\
\hline & & & & & & & $Q$ & $\operatorname{Pr}$ & 0,25 & 1,77 & 1,16 & 0,19 & 0,15 \\
\hline & & & & & & & & $\mathrm{DE}$ & 0,15 & 1,43 & 0,90 & 0,15 & 0,11 \\
\hline & & & & & & & $C$ & $\operatorname{Pr}$ & 0,36 & 0,57 & 0,12 & 0,27 & 0,10 \\
\hline & & & & & & & & $\mathrm{DE}$ & 0,40 & 0,41 & 0,08 & 0,19 & 0,07 \\
\hline
\end{tabular}

CPUE: capturas por unidad de esfuerzo (kg/hp), Pr: promedio, DE: desviación estándar.

PH: período hidrológico, V: vaciante, tC: transición a creciente, C: creciente y tV: transición a vaciante, AC: ambiente acuático, R: río, Q: quebrada, A: caño y C: cocha. CM: comunidades, BV: Buena Vista, CH: El Chino, SP: San Pedro. 

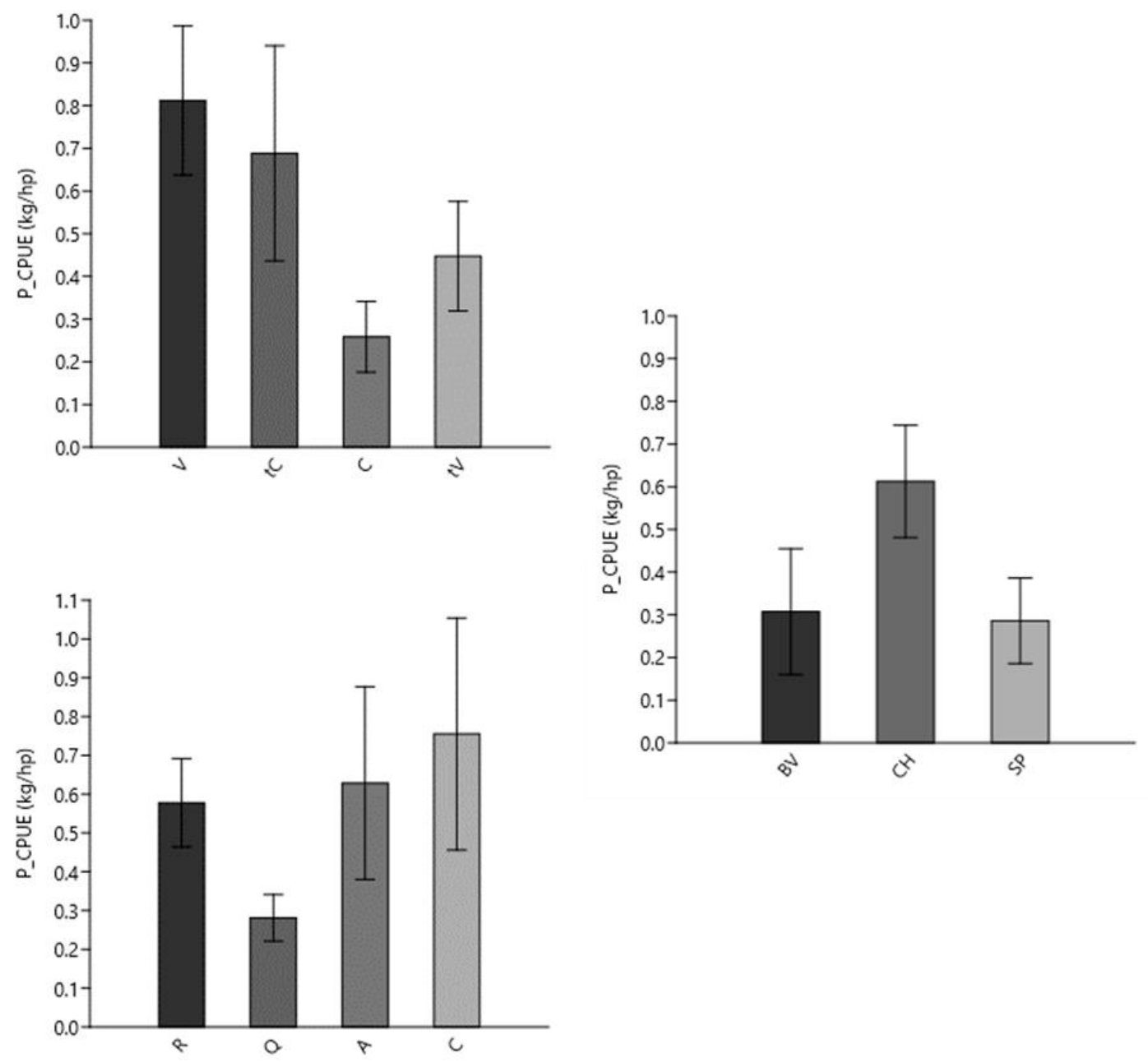

Figura 3. Captura por Unidad de Esfuerzo promedio anual de la pesca en la CRT, considerando período hidrológico ( $\mathrm{V}$ : vaciante, $\mathrm{tC}$ : transición a creciente, C: creciente y $\mathrm{tV}$ : transición a vaciante), ambiente acuático (R: río, Q: quebrada, A: caño y C: cocha) y comunidades (BV: Buena Vista, CH: El Chino, SP: San Pedro). Barras de error: intervalo de confianza a $95 \%$. 

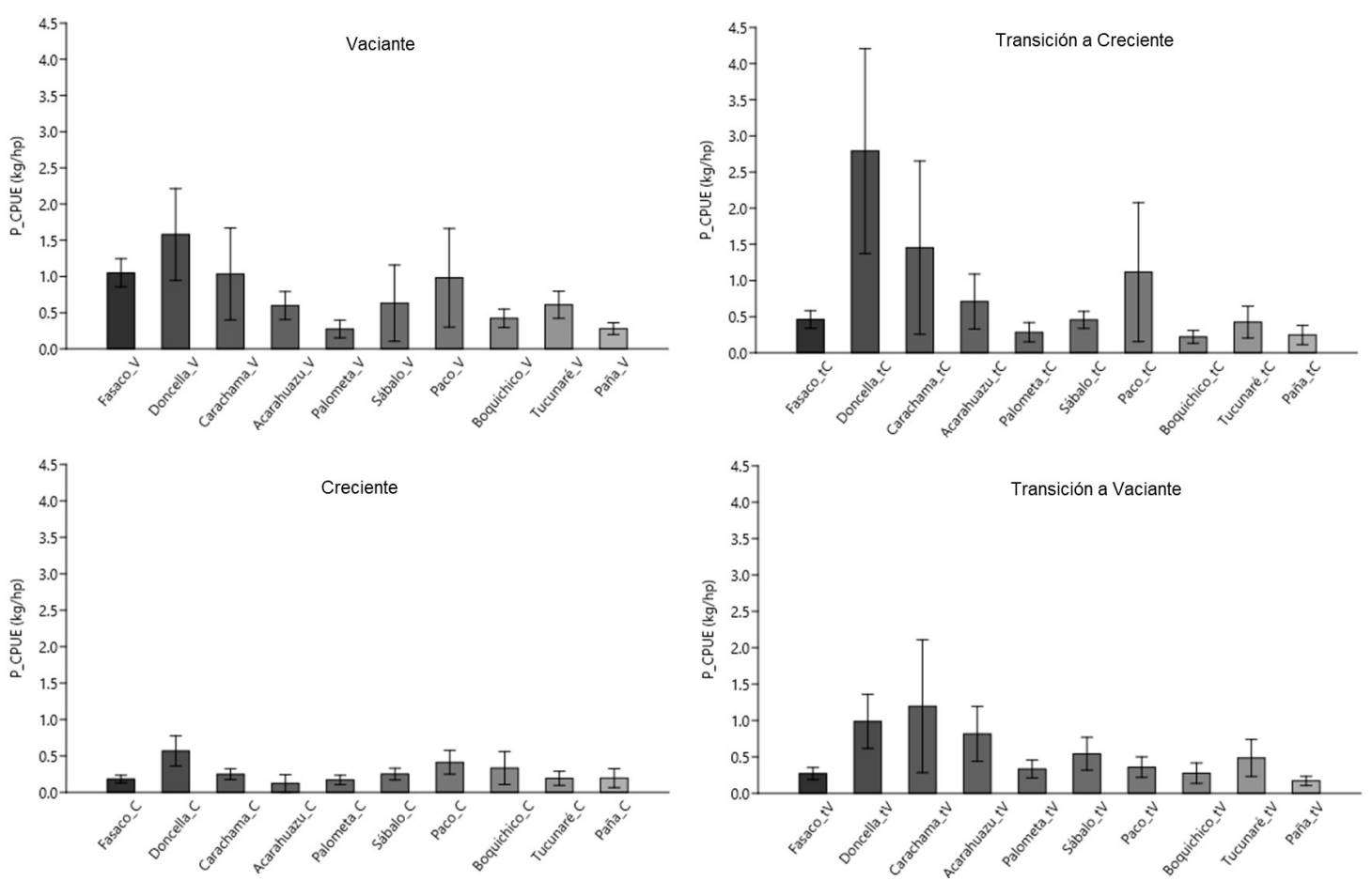

Figura 4. Captura por Unidad de Esfuerzo promedio por especie en la CRT, considerando período hidrológico. Barras de error: intervalo de confianza a $95 \%$.
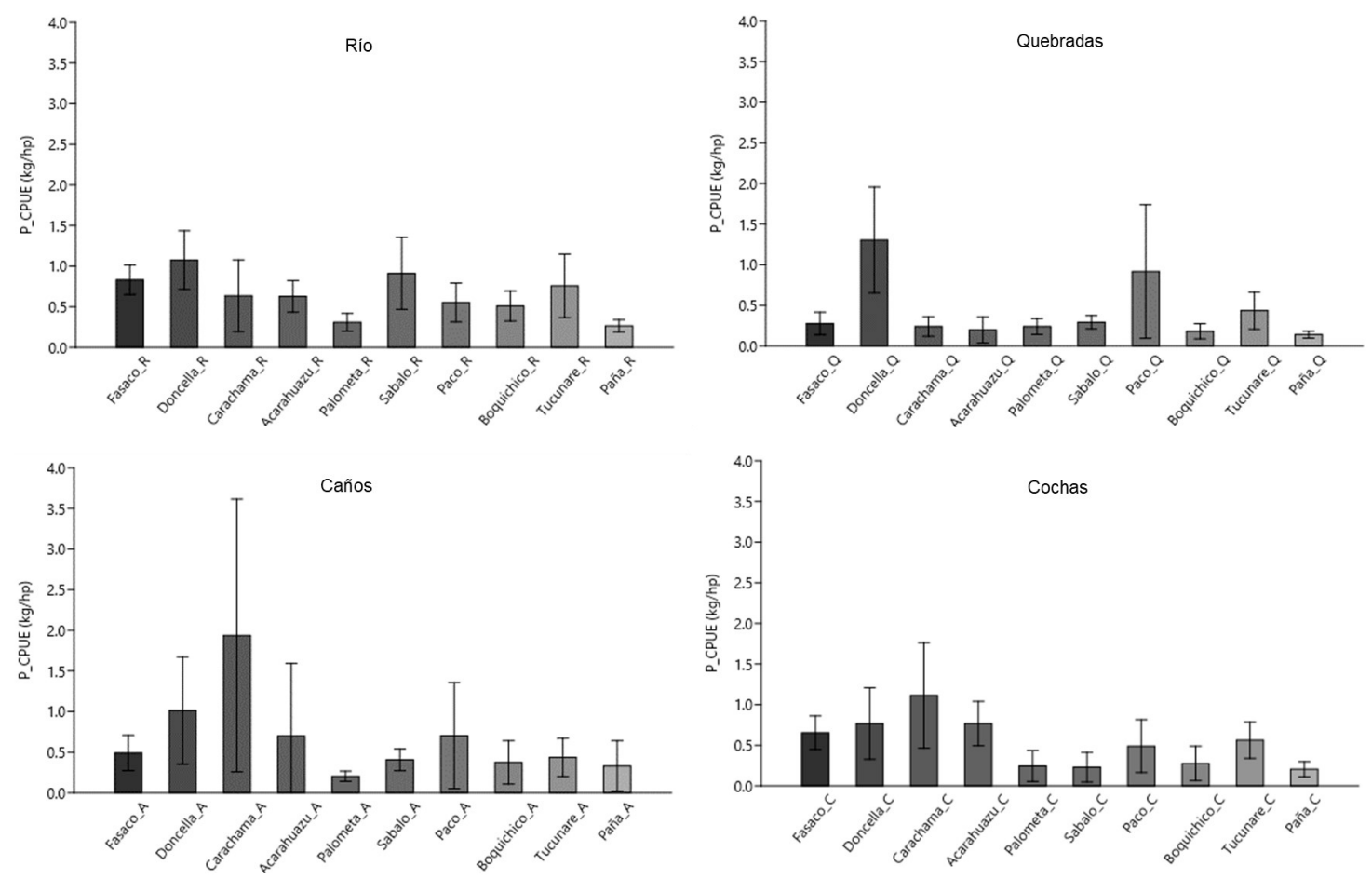

Figura 5. Captura por Unidad de Esfuerzo promedio por especie en la cuenca del río Tahuayo, considerando ambientes acuáticos. Barras de error: intervalo de confianza a $95 \%$. 
Tabla 3. Captura por Unidad de Esfuerzo promedio anual de las principales especies capturadas en la cuenca del río Tahuayo, incluyendo su distribución por comunidad, período hidrológico y ambiente acuático.

\begin{tabular}{|c|c|c|c|c|c|c|c|c|c|c|c|c|c|}
\hline \multirow{3}{*}{ Especies } & \multicolumn{13}{|c|}{ CPUE promedio ( $\mathrm{Pr}, \mathrm{kg} / \mathrm{hp})$ y desviación estándar } \\
\hline & & \multirow{2}{*}{ Anual } & \multicolumn{3}{|c|}{ CM } & \multicolumn{4}{|c|}{$\mathrm{PH}$} & \multicolumn{4}{|c|}{$A C$} \\
\hline & & & BV & $\mathrm{CH}$ & SP & $\mathrm{V}$ & $\mathrm{tC}$ & $\mathrm{C}$ & $\mathrm{tV}$ & $\mathrm{R}$ & Q & A & $\mathrm{C}$ \\
\hline \multirow{2}{*}{ Carachama Chica } & $\operatorname{Pr}$ & 1,66 & 2,32 & 1,36 & - & 5,70 & 0,95 & 0,27 & 0,94 & 0,37 & 0,10 & 0,60 & 2,68 \\
\hline & $\mathrm{DE}$ & 3,41 & 5,56 & 2,02 & - & 5,94 & 1,49 & 0,23 & 2,17 & 0,24 & 0,08 & 0,47 & 4,28 \\
\hline \multirow{2}{*}{ Cunchimama } & $\mathrm{Pr}$ & 1,49 & - & 0,65 & 2,67 & 4,72 & 2,33 & 1,16 & 1,07 & - & 1,51 & 1,25 & - \\
\hline & $\mathrm{DE}$ & 1,20 & - & 0,60 & 0,66 & 1,04 & 1,95 & 1,13 & 0,88 & - & 1,25 & - & - \\
\hline \multirow{2}{*}{ Doncella } & $\mathrm{Pr}$ & 1,26 & 0,86 & 1,21 & 2,25 & 1,58 & 2,79 & 0,57 & 0,99 & 1,08 & 1,30 & 1,01 & 0,77 \\
\hline & $\mathrm{DE}$ & 1,18 & 0,82 & 0,89 & 2,1 & 1,52 & 2,17 & 0,38 & 0,93 & 0,92 & 1,41 & 0,89 & 0,59 \\
\hline \multirow{2}{*}{ Carachama } & $\mathrm{Pr}$ & 1,23 & 0,31 & 1,66 & - & 1,03 & 1,46 & 0,25 & 1,20 & 0,64 & 0,24 & 1,94 & 1,11 \\
\hline & $\mathrm{DE}$ & 2,13 & 0,32 & 2,46 & - & 1,68 & 2,52 & 0,16 & 2,19 & 1,10 & 0,16 & 3,20 & 1,78 \\
\hline \multirow{2}{*}{ Tigre Zungaro } & $\operatorname{Pr}$ & 0,88 & 0,33 & 0,87 & 1,26 & 2,47 & 1,71 & 0,49 & 0,35 & 1,19 & 0,93 & 1,22 & 0,33 \\
\hline & $\mathrm{DE}$ & 0,97 & 0,28 & 0,86 & 1,34 & 1,20 & 1,53 & 0,57 & 0,28 & 1,46 & 0,96 & - & 0,24 \\
\hline \multirow{2}{*}{ Acarahuazu } & $\mathrm{Pr}$ & 0,68 & 0,47 & 0,76 & 0,07 & 0,60 & 0,71 & 0,12 & 0,82 & 0,63 & 0,20 & 0,70 & 0,77 \\
\hline & $\mathrm{DE}$ & 0,64 & 0,42 & 0,68 & 0,08 & 0,52 & 0,73 & 0,15 & 0,90 & 0,49 & 0,20 & 1,29 & 0,76 \\
\hline \multirow{2}{*}{ Paco } & $\mathrm{Pr}$ & 0,65 & 0,33 & 0,54 & 1,98 & 0,98 & 1,12 & 0,41 & 0,36 & 0,55 & 0,92 & 0,70 & 0,49 \\
\hline & $\mathrm{DE}$ & 1,13 & 0,28 & 0,46 & 3,15 & 1,51 & 1,39 & 0,24 & 0,30 & 0,52 & 1,63 & 0,82 & 0,66 \\
\hline \multirow{2}{*}{ Fasaco } & $\mathrm{Pr}$ & 0,54 & 0,44 & 0,62 & 0,35 & 1,05 & 0,46 & 0,18 & 0,27 & 0,83 & 0,28 & 0,49 & 0,65 \\
\hline & $\mathrm{DE}$ & 0,50 & 0,32 & 0,54 & 0,53 & 0,71 & 0,31 & 0,14 & 0,27 & 0,59 & 0,38 & 0,54 & 0,68 \\
\hline \multirow{2}{*}{ Tucunare } & $\mathrm{Pr}$ & 0,51 & 0,59 & 0,50 & 0,45 & 0,61 & 0,42 & 0,19 & 0,49 & 0,76 & 0,43 & 0,44 & 0,56 \\
\hline & $\mathrm{DE}$ & 0,44 & 0,31 & 0,48 & 0,44 & 0,51 & 0,32 & 0,15 & 0,57 & 0,96 & 0,44 & 0,36 & 0,51 \\
\hline \multirow{2}{*}{ Sabalo* } & $\mathrm{Pr}$ & 0,48 & 0,40 & 0,58 & 0,16 & 0,63 & 0,45 & 0,25 & 0,54 & 0,91 & 0,29 & 0,41 & 0,23 \\
\hline & $\mathrm{DE}$ & 0,58 & 0,43 & 0,67 & 0,06 & 0,97 & 0,8 & 0,21 & 0,67 & 1,13 & 0,22 & 0,25 & 0,30 \\
\hline \multirow{2}{*}{ Corvina } & $\mathrm{Pr}$ & 0,48 & 0,44 & 0,60 & 0,17 & 0,31 & 0,38 & 0,28 & 0,60 & 0,43 & 0,51 & 0,06 & 0,13 \\
\hline & $\mathrm{DE}$ & 1,05 & 0,35 & 1,27 & 0,18 & 0,36 & 0,40 & 0,33 & 1,33 & 0,35 & 1,17 & - & 0,08 \\
\hline \multirow{2}{*}{ Shuyo } & $\mathrm{Pr}$ & 0,46 & 0,08 & 0,62 & 0,40 & 0,43 & 0,53 & 0,25 & 0,43 & 0,43 & 0,20 & 0,58 & 0,36 \\
\hline & $\mathrm{DE}$ & 0,81 & 0,04 & 0,94 & 0,63 & 0,49 & 0,68 & 0,42 & 0,93 & 0,73 & 0,32 & 1,28 & 0,39 \\
\hline \multirow{2}{*}{ Bujurqui } & $\mathrm{Pr}$ & 0,35 & 0,23 & 0,42 & 0,15 & 0,42 & 0,35 & 0,19 & 0,25 & 0,32 & 0,28 & 0,32 & 0,62 \\
\hline & $\mathrm{DE}$ & 0,46 & 0,28 & 0,52 & 0,18 & 0,50 & 0,35 & 0,19 & 0,33 & 0,44 & 0,49 & 0,24 & 0,97 \\
\hline \multirow{2}{*}{ Boquichico } & $\mathrm{Pr}$ & 0,29 & 0,18 & 0,35 & 0,19 & 0,42 & 0,22 & 0,33 & 0,28 & 0,51 & 0,18 & 0,37 & 0,28 \\
\hline & $\mathrm{DE}$ & 0,33 & 0,15 & 0,39 & 0,18 & 0,42 & 0,21 & 0,49 & 0,41 & 0,56 & 0,22 & 0,64 & 0,55 \\
\hline \multirow{2}{*}{ Palometa } & $\mathrm{Pr}$ & 0,27 & 0,22 & 0,34 & 0,09 & 0,27 & 0,28 & 0,17 & 0,33 & 0,31 & 0,24 & 0,20 & 0,24 \\
\hline & DE & 0,29 & 0,21 & 0,33 & 0,04 & 0,29 & 0,26 & 0,18 & 0,38 & 0,29 & 0,28 & 0,12 & 0,34 \\
\hline \multirow{2}{*}{ Lisa* } & $\mathrm{Pr}$ & 0,24 & 0,24 & 0,25 & 0,22 & 0,35 & 0,25 & 0,13 & 0,20 & 0,39 & 0,18 & 0,35 & 0,12 \\
\hline & $\mathrm{DE}$ & 0,24 & 0,27 & 0,25 & 0,19 & 0,28 & 0,24 & 0,14 & 0,30 & 0,36 & 0,19 & 0,42 & 0,13 \\
\hline \multirow{2}{*}{ Paña* } & $\mathrm{Pr}$ & 0,19 & 0,15 & 0,24 & 0,11 & 0,28 & 0,25 & 0,19 & 0,17 & 0,27 & 0,14 & 0,33 & 0,21 \\
\hline & DE & 0,19 & 0,14 & 0,23 & 0,05 & 0,23 & 0,26 & 0,32 & 0,18 & 0,20 & 0,11 & 0,52 & 0,24 \\
\hline
\end{tabular}

CPUE: capturas por unidad de esfuerzo (kg/hp), Pr: promedio, DE: desviación estándar

CM: comunidades, BV: Buena Vista, CH: El Chino, SP: San Pedro, PH: período hidrológico, V: vaciante, tC: transición a creciente, C: creciente y $\mathrm{tV}$ : transición a vaciante, $\mathrm{AC}$ : ambiente acuático, R: río, Q: quebrada, $\mathrm{A}$ : caño y C: cocha. CM: comunidades, BV: Buena Vista, CH: El Chino, SP: San Pedro.

*Nombre local que agrupa varias especies 
La variación de las CPUE por periodos hidrológicos obtenido para la CRT (Tabla 2) presentó un patrón similar al encontrado en otros tributarios menores en la región, como en la cuenca del río Napo (García et al., 2009). Los mayores valores obtenidos en los meses de vaciante están asociados a una reducción de los ambientes acuáticos, por tanto, una mayor concentración del recurso pesquero y mayor probabilidad de captura de los peces a los aparejos de pesca (Junk et al., 2015; Endo et al., 2016). Esto se vio reflejado en el promedio de tiempo efectivo de pesca invertido (V 4,2 horas $\mathrm{DE}=2,5$; $\mathrm{C} 11,3$ horas $\mathrm{DE}=4,7$ ), dada la mayor dispersión del recurso pesquero para la temporada de aguas altas. Los valores de CPUE en los meses de transición a creciente están asociados al movimiento del recurso pesquero hacia el bosque inundable, sus patrones de migración determinan la estacionalidad de sus capturas en cuencas del bosque inundable, como el río Tahuayo, y han sido observados en otras regiones de la Amazonía (Goulding, 1980; Cerdeira et al., 2000).

La poca variabilidad de las CPUE entre los diferentes hábitats en la CRT (Tabla 2 y 3 ) evidencia un ecosistema cuyo recurso pesquero está asociado a la disponibilidad y acceso a diferentes espacios que les proveen alimento, refugio y condiciones para reproducción (Goulding, 1980; Oliveira et al., 2006; Correa et al., 2008; Lobón-Cerviá et al., 2015) a lo largo del ciclo hidrológico. En la CRT, especies depredadoras como el fasaco y los grandes bagres (principalmente la doncella) utilizan los cuatro tipos de hábitats, según la disponibilidad de alimento (peces de pequeño y mediano porte), principalmente en hábitats de bosque inundable y aguas abiertas (Goulding, 1980; Cerdeira et al., 2000; Saint-Paul et al., 2000). La alta capturabilidad de las carachamas en vaciante en las cochas y caños de la CRT está asociada a las condiciones de poco flujo en estos hábitats, espacios ideales para el acúmulo de materia orgánica en el fondo, base de la alimentación de estas especies sedentarias, y donde también encuentran espacios de reproducción (Junk et al., 1989; Cerdeira et al., 2000).

Las mayores CPUE de peces para el río Tahuayo se asocian a especies de mayor porte, como los grandes bagres, principalmente en vaciante y las temporadas de transición, lo cual evidencia su rol como vía principal en el tránsito de peces entre el río Amazonas y el resto de los hábitats ubicados dentro de la cuenca (Goulding, 1980; Cerdeira et al., 2000). Dentro de las quebradas destaca la Quebrada Blanco, tributario con alta carga de sedimentos que proviene del bosque de altura de la cuenca y descarga en el río Tahuayo (Coomes, 1995). Esta quebrada es utilizada principalmente por los pescadores de San Pedro (todo el año) y por los pescadores de El Chino (creciente y transición a creciente). Especies de gran porte como los grandes bagres, paco y sábalos destacan por su alta capturabilidad en esta quebrada, lo cual la convierte, después del río Tahuayo, en una de las principales zonas de pesca en esta cuenca.

Todosestosresultadosmuestranlaimportancia de considerar las dinámicas pesqueras de pequeña escala, bajo un enfoque de cuenca que considera diferentes ambientes acuáticos y sus dinámicas a lo largo del ciclo hidrológico. Esto cobra vital importancia cuando se reconoce que la principal amenaza al mantenimiento de los stocks pesqueros que utilizan el bosque inundable no es la sobrepesca, sino la pérdida de hábitats (Crampton et al., 2004; Arantes et al., 2019). Estas características de la pesca de pequeña escala, así como el conocimiento que tienen los pescadores sobre la dinámica de los bosques inundables, representan las bases para promover un manejo participativo efectivo. Sin embargo, en la actual reglamentación de la pesca en la Amazonía peruana no se visualiza este tipo 
de pesca realizada por comunidades ribereñas ampliamente distribuidas, que basan su subsistencia en el aprovechamiento de una gran variedad de recursos naturales, considerando dinámicas ecológicas y escalas espaciales y temporales específicos (Mahon, 1997; Pauly, 1997; Berkes et al., 2001; Castello et al., 2013). Un manejo pesquero que incluya la pesca de pequeña escala, debe considerar medidas basadas en una zonificación de hábitats, que considere los cambios estacionales y la diversidad de especies, y deben complementarse con acciones de control y vigilancia, como las establecidas por las comunidades del Tahuayo (Smith et al., 2001; Chirif, 2012). Para el caso de la CRT se propone que la pesca en los ríos y quebradas (Tahuayo y Blanco) se enfoque a especies de mayor porte, considerando los sucesos migratorios de estas especies y regulando el uso de aparejos de pesca (tamaño de abertura de malla estirada; número y cantidad de anzuelos). Asimismo, la pesca en las cochas y caños durante los meses de vaciante debe controlar el esfuerzo pesquero (horas de pesca). Se debe monitorear las poblaciones de especies importantes, como la carachama negra, determinando sus periodos de reproducción, talla mínima y esfuerzos máximos de captura. Esta iniciativa, que ha contado con la participación activa de los pescadores, debe replicarse en otras cuencas similares de Loreto, con una metodología estandarizada, cuya información permita actualizar de la manera más adecuada las regulaciones pesqueras en la Amazonía peruana.

\section{AGRADECIMIENTOS}

Agradecemos de manera especial a los pescadores de las comunidades Buena Vista, El Chino y San Pedro, a la directiva de la Asociación de Pescadores Artesanales Puma Garza I, y al Comité de Gestión del Área de Conservación Regional Tamshiyacu Tahuayo (ACRCTT), por el apoyo durante el monitoreo de la pesca comunitaria. Al museo de Museo de Historia Natural de la Universidad Nacional Mayor de San Marcos por su apoyo en la identificación de especies. A WCS, en especial a Paola Matayoshi, Armando Mercado, Mariana Montoya y Sandra Ríos por los aportes al manuscrito. Este trabajo ha sido posible gracias al apoyo financiero de la Fundación Gordon \& Betty Moore y The Arcadia Charitable Trust.

\section{REFERENCIAS BIBLIOGRÁFICAS}

ACRCTT. 2010. Plan Maestro del Área de Conservación Regional Comunal Tamshiyacu Tahuayo. Gobierno Regional de Loreto y Programa de Conservación, Gestión y Uso Sostenible de la Diversidad Biológica de Loreto - PROCREL, Iquitos. 138pp.

Almeida, O.; Lorenze, K.; McGrath, D.G. 2009. Fishing agreements in the lower Amazon: for gain and restraint. Fisheries Management and Ecology, 16: 61-67. DOI: https://doi. org/10.1111/j.1365-2400.2008.00647.x

Anderson, E.; Montoya, M.; Soto, A.; Flores, H. 2009. Challenges and opportunities for comanagement of a migratory fish (Prochilodus nigricans) in the peruvian Amazon. En: Haro, A.J.; Smith, K.L.; Rulifson, R.A.; Moffitt, C.M.; Klauda, R.J.; Dadswell, M.J.; Cunjak, R.A.; Cooper, J.E.; Beal, K.L.; Avery, T.S. (Eds). Challenges for diadromous fishes in a dynamic global environment. Bethesda, MD: American Fisheries Society, Symposium 69. p. 1-16.

Arantes, C.C.; Winemiller, K.O.; Asher, A.; Castello, L.; Hess, L.; Petrere Jr., M.; Freitas, C.E.C. 2019. Flooplain land cover affects biomas distribution of fish functional diversity in the Amazon River. Scientific Reports, 9: 16684. DOI: https://doi.org/10.1038/s41598-01952243-0

Berkes, F.; Mahon, R.; McConney, P.; Pollnac, R.; Pomeroy, R. 2001. Managing small-scale 
fisheries. Alternative directions and methods. International Development Research Centre, Otawa, Canadá. 308pp.

Berkes, F. 2008. La pesquería de pequeña escala: alternativas al manejo convencional de recursos. En: Pinedo D.; Soria, C. (Eds). El Manejo de las pesquerías en ríos tropicales de Sudamérica. Instituto del Bien Común. p. 443459.

Cañas C. 2000. La pesca en la provincia de Tambopata: monitoreo y ordenamiento pesquero en el sureste peruano. En: Rodriguez, L.O. (Ed). El Manu y otras experiencias de Investigaciónymanejo de bosquesneotropicales. p. 178-186.

Castello, L.; McGrath, D.C.; Arantes, C.C.; Almeida, O.T. 2013. Heterogeneity in small-scale fisheries management: The Amazon case. Marine Policy, 38: 557-565. DOI: https://doi. org/10.1016/j.marpol.2012.09.001

Cerdeira, R.G.P.; Ruffino, M.; Isaac, V.J. 2000. Fish catches among riverside communities around Lago Grande de Monte Alegre, Lower Amazon, Brazil. Fisheries Management and Ecology, 7:355-374. DOI: https://doi.org/10.1046/ j.1365-2400.2000.007004355.x

Chirif, A. 2012. La historia del Tahuayo contada por sus moradores. Wildlife Conservation Society, Lima, Perú. 99pp.

Coomes, O.T. 1992. Making a living in the Amazon rain forest: Peasants, land, and economy in the Tahuayo river basin of northeastern Peru. Tesis de doctorado, University of WisconsinMadison, EE UU. 415pp.

Coomes, 0.T. 1995. A century of rain forest use in western Amazonia. Lessons for extractionbased conservation of tropical forest resources. Forest \& Conservation History, 39(3): 108-120. DOI: http://dx.doi.org/10.2307/3983515

Correa, S.B.; Crampton, W.G.R.; Chapman, L.J.; Albert, J.S. 2008. A comparison of flooded forest and floating meadow fish assemblages in an upper Amazon floodplain. Journal of Fish Biology, 72: 629-644. DOI: https://doi. org/10.1111/j.1095-8649.2007.01752.x

Crampton, W.G.R.; Castello, L.; Viana, J.P. 2004. Historical trends, current status, and factors affecting sustainability. En: Silvius, K.M; Bodmer, R.E.;Fragoso, J.M.V. 2004. People in nature: Wildlife conservation in South and Central America. Columbia University Press, New York, USA, p. 76-122.

DIREPRO-L. 2016. Boletín Informes mensuales. Dirección de Extracción y Procesamiento Pesquero y Direcciones Sub-Regionales y Oficinas de la Producción. Oficina de Planeamiento y Presupuesto. Direpro-Loreto. Iquitos.

DIREPRO-L. 2017. Boletín Informes mensuales. Dirección de Extracción y Procesamiento Pesquero y Direcciones Sub-Regionales y Oficinas de la Producción. Oficina de Planeamiento y Presupuesto. Direpro-Loreto. Iquitos.

DIREPRO-L. 2019. Base de datos de desembarque pesquero de consumo humano directo. Oficina de Planeamiento y Presupuesto. DireproLoreto. Iquitos.

Endo, W.; Peres, C.A.; Haugaasen, T. 2016. Flood pulse dynamics affects exploitation of both aquatic and terrestrial prey by Amazonian floodplain settlements. Biological Conservation, 201: 129-136. DOI: https://doi. org/10.1016/j.biocon.2016.07.006

Espinoza, J.C.; Guyot, J.L.; Ronchail, J.; Cochonneau, G.; Filizola, N.; Fraizy, P.; Labat, D.; de Oliveira, E.; Ordoñez, J.J.; Vauchel, P. 2009. Contrasting regional discharge evolutions in the Amazon Basin (1974-2004). Journal of Hydrology, 375: 297-311. DOI: https://doi.org/10.1016/j. jhydrol.2009.03.004

Flores, F; Antúnez, M; Padilla, G; Silva, M; Lehm, Z. 2016. Evaluación de fortalezas y debilidades de la gobernanza en la región del Área de 
Conservación Regional Tamshiyacu - Tahuayo. Informe técnico. WCS, Iquitos. 159pp.

García, A.; Vargas, G.; Tello, S.; Duponcelle, F. 2012. Desembarque de pescado fresco en la ciudad de Iquitos, Región Loreto - Amazonía peruana. Folia Amazónica, 21: 45-52. DOI: https://doi. org/10.24841/fa.v21i1-2.31

García, A.; Tello, S.; Vargas, G.; Duponchelle, F. 2009. Patterns of commercial fish landing in the Loreto Region (Peruvian Amazon) between 1984 and 2006. Fish Physiology and Biochemistry. DOI https://doi.org/10.1007/ s10695-008-9212-7

García-Dávila, C.; Sánchez, H.; Flores, M.; Mejia, J.; Angulo, C.; Castro-Ruiz, D.; Estivals, G.; García, A.; Vargas, G.; Nolorbe, C.; Núñez, J.; Mariac, C.; Duponchelle, F.; Renno, J.F. 2018. Peces de consumo de la Amazonía Peruana. Instituto de Investigaciones de la Amazonía Peruana (IIAP). Iquitos. 218 pp.

Goulding, M. 1980. The fishes and the forest: Explorations in amazonian natural history. University of California Press, Berkeley. 280pp. Hammer, Ø.; Harper, D.A.T.; Ryan, P.D. 2001. PAST: Paleontological statistics software package for education and data analysis. Palaeontologia Electronica, 4(1):1-9.

Junk, W.J.; Wittmann, F.; Schongart, J.; Piedade, M.T.F. 2015. A classification of the major habitats of amazonian black-water river floodplains and a comparison with their whitewater counterparts. Wetlands Ecology and Management, 23(4): 677-693. DOI: https:// doi.org/10.1007/s11273-015-9412-8

Junk, W.; Bayley, P.B.; Sparks, R.E. 1989. The flood pulse concept in rIver floodplain systems. Can. Spec. Publ. Fish. Aquat. Sci. 106: 110-127.

Lobón-Cerviá, J.; Hess, L.L.; Melack, J.M.; AraujoLima, C.A.R.M. 2015. The importance of forest cover for fish richness and abundance on the Amazon floodplains. Hydrobiologia, 750: 245-
255. DOI: https://doi.org/10.1007/s10750014-2040-0

McDaniels, J.M. 1997. Communal fisheries management in the Peruvian Amazon, Human Organization, 56 (2):147-152.

Mahon, R. 1997. Does fisheries science serve the needs of managers of small stocks in developing countries? Canadian Journal of Fisheries and Aquatic Sciences, 54: 2207-2213. Marina de Guerra del Perú. 2018. Avisos a los navegantes fluviales: edición noviembre 2018. Servicio de Hidrografía y Navegación de la Amazonía, Iquitos. 40pp.

Oliveira, A.C.B.; Martinelli, L.A.; Moreira, M.Z.; Soares, M.G.M.; Cyrino, J.E.P. 2006. Seasonality of energy sources of Colossoma macropomum in a floodplain lake in the Amazon - Lake Camaleao, Amazonas, Brasil. Fisheries Management and Ecology, 13: 135142. DOI: https://doi.org/10.1111/j.13652400.2006.00481.x

Ortega, H; Hidalgo, M.; Correa, E.; Espino, J.; Chocano, L.; Trevejo, G.; Meza, V.; Cortijo, A.M.; Quispe. R. 2011. Lista anotada de peces de aguas continentales del Perú. Estado actual del conocimiento, distribución, usos y aspectos de conservación. Ministerio del Ambiente, Dirección General de Diversidad Biológica - Museo de Historia Natural, UNMSM, Lima. 48pp.

Pauly, D. 1997. Small-Scale fisheries in the tropics: Marginality, marginalization, and some implications for fisheries management, En: Pikitch, E.K.; Huppert, D.D.; Sissenwine, M.P. (Eds). Global trends: Fisheries management. American Fisheries Society - Symposium 20, Bethesda, Maryland, p. 40-49.

Riofrío, J.C.; Ferré Rodriguez, W.R.; Velarde Ríos, D.A. 2008. Contribuciones para el manejo de la pesquería comercial en Pucallpa (Ucayali, Perú). En: Pinedo, D.; Soria, C. (Eds). El manejo de las pesquerías en los ríos tropicales de 
Sudamérica. Centro de Investigaciones para el Desarrollo, p. 171-188.

Rodríguez, V.; Castro, E.; Collado, E. 2018. Gobernanza colaborativa en la pesca: Modelo participativo para la gestión pesquera en la Amazonia peruana. Instituto del Bien Común, Lima, Perú. 318pp.

Saint-Paul, U.; Zuanon, J.; Villacorta-Correa, M.A.; García,M.; Fabré, N.N.; Berger,U.; Junk,W.J. 2000. Fish communities in central Amazonia whiteand blackwater floodplains. Environmental Biology of fishes, 57: 235-250. DOI: https:// doi.org/10.1023/A:1007699130333

Smith, R.C.; Pinedo, D.; Summers P.M.; Almeyda A. 2001. Tropical rhythms and collective action. Community-based fisheries management in the face of amazonian unpredictability. IDS Bulletin, 32(4): 36-46. DOI: https://doi.org/ 10.1111/j.1759-5436.2001.mp32004005.x Summers, P.M.; Pinedo D. 2002. Bienes comunes enraizados: descripción densa de dos casos de manejo comunitario en la Amazonía peruana. Instituto del Bien Común, Instituto de Pesquisa Ambiental da Amazonia, Oxfam América, Woods Hole Research Center, Iquitos.148pp.

Vargas, M. 2017. Monitoreo de la actividad pesquera en 03 comunidades ubicadas en la zona de amortiguamiento del ACR CRTT. WCS, Iquitos. 75pp.

Wildlife Conservation Society. 2020. Evaluación espacial de los principales conflictos pesqueros en la región Loreto. WCS, Iquitos. 88pp.

Recibido: 17 de octubre de 2020 Aceptado para publicación: 22 de diciembre de 2020

Esta obra está bajo una Licencia Creative Commons Atribución-NoComercial-SinDerivar 4.0 Internacional. 